\title{
Ultrastructural Characterization of Corpus Cavernosum of Ageing, Orchidectomy and Diabetes Rat Experimental Models
}

\author{
A. L. Cordeiro*, A. Figueiredo ", F. Godinho*, I. Martins ${ }^{*}$, P. Vendeira ${ }^{* *}$, H. Almeida ${ }^{*}$ and D. \\ Neves * \\ * Laboratory of Molecular Cell Biology of Faculty of Medicine and IBMC of Universidade do \\ Porto - 4200-319 Porto - Portugal; \\ ** Department of Urology of S. João Central Hospital, Porto - 4200-319 Porto - Portugal. The \\ first 4 authors had the same contribution to the presented work \\ delmagal@med.up.pt
}

Small vessels of the penis are very sensitive to structural and functional changes, and small disturbances can conduce to any degree of erectile dysfunction (ED) [1]. For this motive, ED is now considered as an early manifestation of atherosclerosis and consequently a precursor of systemic vascular disease [2]. Ageing, diabetes and hipogonadic states are recognized risk factors for ED, contributing together to vascular damage of penile tissue. Penile trabeculae of corpus cavernosum in the rat, constituted by connective tissue with abundant collagen fibrils, scarce fibroblasts and fibroelastic components, support smooth muscle fibers in subendothelium location. Corpus cavernosum structural elements act in concert, allowing increase of intra-cavernous arterial flow and smooth muscle relaxing, processes which are fundamental to penis erection. The purpose of this study is to clarify ultrastructural organization of corpus cavernosum of experimental rat models of known ED-risk factors.

Male Wistar rats were divided in experimental groups $(n=6)$, of different ages $(2,6,12,18$ and $24 \mathrm{mo}$ ), orchidectomized at $2 \mathrm{mo}$ and sacrificed at $6 \mathrm{mo}$, and diabetics (streptozotocin $90 \mathrm{mg} / \mathrm{kg}$ ip injection at $2 \mathrm{mo}$ and sacrificed at $6 \mathrm{mo}$ ). Androgen depletion in serum of orchidectomized rats was revealed by RIA. Penis fragments were removed, fixed in glutaraldehyde, processed to Epon embedding and observed by transmission electron microscopy (Jeol 100B) for ultrastructural study. Morphometric study was performed in random selected 16 micrographs. Total areas of endothelium, smooth muscle, collagen, lipids and connective tissue cells were quantified.

Aged (18m and 24m), diabetic and orchidectomized rats presented signals of vascular damage, disorganized fibers of collagen, diminished smooth muscle layer and loss of endothelium integrity were observed, in contrast to younger animals. Foam cells which are atherosclerosis precursors were also seen in corpus cavernosum of aged and orchidectomized rats. Morphometric analysis demonstrated that ageing, androgen depletion and diabetes contribute to ED progression by modifying cellular and extracellular matrix organization of cavernosum tissue. In brief, we can conclude that the rat models here characterized are adequated to use as experimental models for ED studies.

\section{References:}

[1] C. Kaya et al., Int. J. Impot. Res.18 (2006)55

[2] C.M.D. Cheitlin, J. Am. Coll. Cardiol. 43 (2004)185

[3] This work was supported by the program Investigação na pré-graduação Caixa Geral de

Depósitos /Universidade do Porto. 


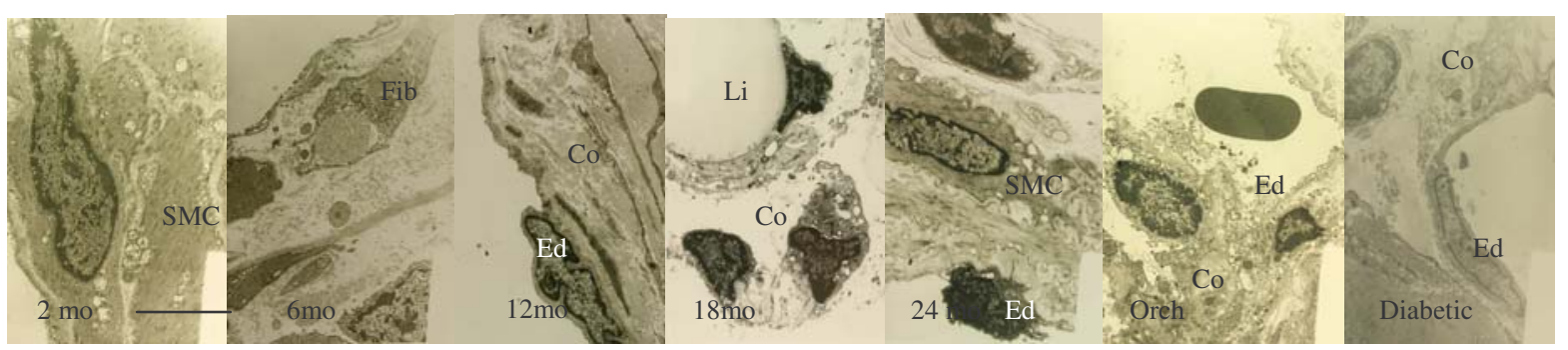

Fig. 1. Corpus cavernosum micrographs from experimental models of ageing $(2,6,12,18$ and 24 months), orchidectomized and diabetic rats. Note smooth muscle cells (SMC), endothelium (Ed), lipid droplets (Li) in cells of aged and orchidectomized rats and collagen (Co) deposition in extracellular matrix. Scale bar - $5 \mu \mathrm{m}$

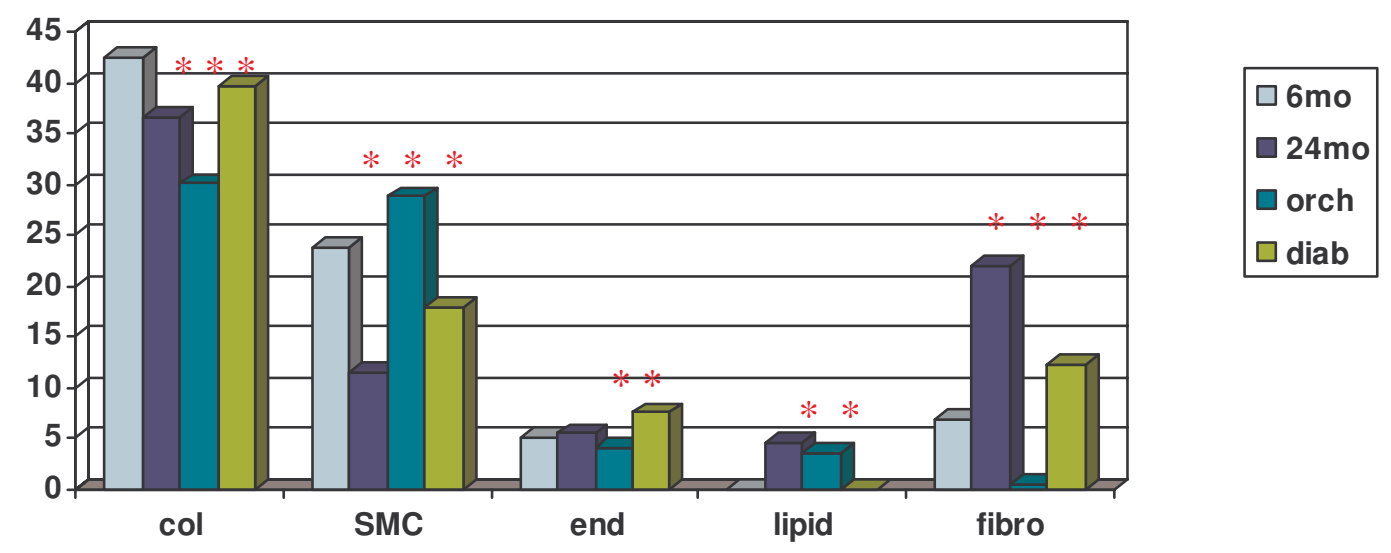

Fig.2. Comparative percentage distribution of cellular constitution of corpus cavernosum in aged (24 mo), orchidectomized and diabetic rat experimental models. Smooth muscle cells (SMC), endothelium (end), fibroblasts (fibro), collagen (col) and lipids. * significant variation when compared with 6 months aged controls $\mathrm{P}<0.02$

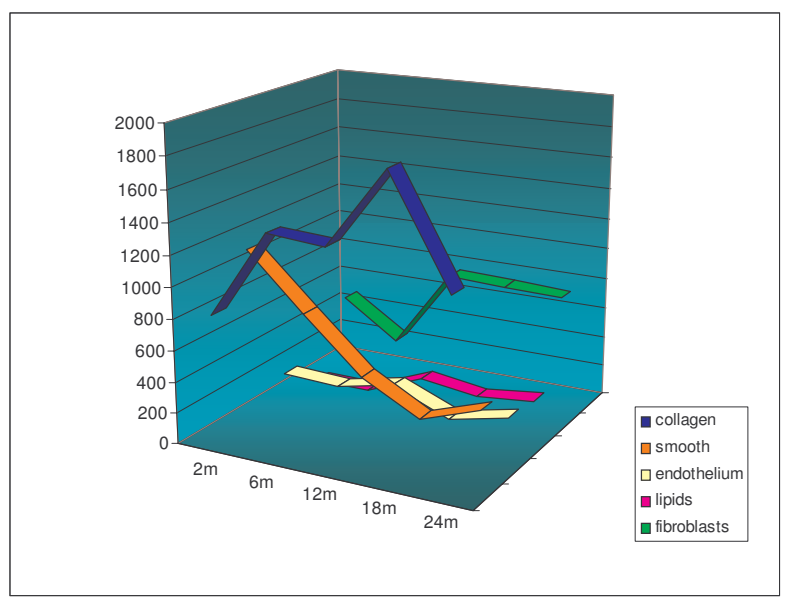

Fig.3. Comparative distribution of cellular constitution of corpus cavernosum during ageing. 